\title{
The sensitivity of MONK calculations to nuclear data parameters
}

\author{
Simon Connolly ${ }^{1, a}$, Christopher Dean ${ }^{2}$, and Ray Perry ${ }^{2}$ \\ 1 British Nuclear Group Sellafield Ltd, Sellafield, Seascale, Cumbria, CA20 1PG, UK \\ 2 Serco Assurance, Winfrith Technology Centre, Winfrith, Dorchester, Dorset, DT2 8DH, UK
}

\begin{abstract}
This paper examines the sensitivity of the MONK criticality code to a variety of nuclear data parameters. The number of equiprobable bins for both secondary energies and secondary angles are varied; the effect of varying the background cross section employed by the sub-group representation in the unresolved region is examined; and variations in cross section resonance reconstruction tolerances are considered. To validate these options we use integral data including results from the GODIVA, JEZEBEL and ZEBRA-8H experiments. The results from this study will be used to optimise data used in the MONK criticality code.
\end{abstract}

\section{Introduction}

MONK [1] is a well-established Monte Carlo code for the assessment of nuclear criticality safety problems, with over thirty-five years of successful application across the whole of the nuclear fuel cycle. Over the last decade, the development of MONK has been performed by a collaboration comprising British Nuclear Group Sellafield Ltd and Serco Assurance, with the MONK code being distributed and maintained in use by Serco Assurance's ANSWERS Software Service.

Over the last few years, a substantial programme of regeneration and renewal has been in progress, to update and enhance various aspects of the MONK methods, data and software. MONK8 [2] was the culmination of geometric improvements, leading to the unification of two previously separate versions of the code (one for criticality, another for reactor physics). Another major strand, in the current production version of the code MONK9 [3], has been the development of a completely new neutron collision processing system and associated nuclear data library [4]. This new collision processor is named BINGO and is designed eventually to replace the current DICE [5] collision processor.

This paper examines the sensitivity of MONK to a variety of nuclear data parameters. The number of equiprobable sampling bins for both secondary angles and energies are varied; the effect of varying the background cross section employed by the sub-group representation in the unresolved region is examined; and variations in cross section resonance reconstruction tolerances are considered.

\section{Description of the BINGO code}

DICE dates from the 1960's and was originally written to use the United Kingdom Nuclear Data Library (UKNDL) [6] and was adapted for use with ENDF-6 [7] structured libraries at a later date. From the outset, BINGO has been designed to

\footnotetext{
a Presenting author,

e-mail: simon.m.connolly@briti shnucleargroup.com
}

use data libraries in the ENDF-6 format, including JEFF3.1 $[8,9]$. To take advantage of modern, modular programming techniques, all coding is in Fortran-90 [10]. There are two parts to the code: the pre-processor and the collision processor.

The pre-processor takes the ENDF-6 library file and converts these data into a form suitable for Monte Carlo sampling. All available neutron formats are processed by the code and library data can be generated at elevated temperatures. Cross section data are represented on a point-wise grid but a subgrouping representation is applied in the unresolved resonance region (URR). Cross section reconstruction, broadening, etc., are handled by NJOY99 $[11,12]$, which is run as a stand-alone code by the pre-processor. New Fortran-90 routines in the preprocessor consider emitted particles.

The BINGO neutron collision processor is an internal module of the MONK criticality code. It accesses the library data created by the pre-processor and samples these data to determine the outcome of collisions, the mean free paths and the secondary angles and energies, etc.

The methods used by the pre-processor are described below.

\subsection{Cross section representation}

Cross sections are stored in the BINGO library as point values on an energy grid chosen so that intermediate values are adequately represented by linear interpolation. A different energy grid is used for each nuclide. This allows cross section variations to be represented accurately in the resolved resonance regions for individual nuclides, while reducing the amount of data needed to represent smooth cross sections.

\subsection{Unresolved resonance region}

A subgroup method is used to treat resonance self-shielding effects in the unresolved region. It is a 2-subgroup technique, in which the cross section in a narrow energy interval is represented as having two possible values. These values are calculated so that they produce the correct average cross section in the interval when the nuclide is present at "infinite 
dilution" and at one other chosen dilution. The aim is to ensure that these cross sections are stored just like normal cross sections over appropriate subdivisions of the interval, so that no special sampling procedure is required. This technique is similar to the one used in DICE, but has been adapted for use with point-energy cross sections and its accuracy has been improved by allowing the subgroups to be of unequal width.

\subsection{Temperature treatment}

For any given nuclide, a BINGO library may contain cross sections for a number of discrete temperatures. In practice, the BINGO libraries provide multi-temperature data for a set of the most important nuclides, while data for other nuclides are at "room temperature" only. It is assumed that the energy and angular data are not temperature dependent, except at thermal energies. Thus, the BINGO library files at elevated temperatures contain only the cross section data (together with thermal scattering data in the case of bound atoms), and the code automatically refers to the room temperature file for the secondary distributions. BINGO does not interpolate between temperature, rather MONK selects data for the nearest available temperature.

\subsection{Delayed neutron data}

The BINGO library includes data for delayed neutrons from fission. These will allow the BINGO collision processor to be used for kinetics applications. In static calculations, the presence of the delayed neutron data allows the correct spectra to be used for delayed neutron data in the BINGO library.

\subsection{Thermal neutron scattering}

For thermal neutron scattering by bound atoms, the data preprocessing is carried out by the BINGO pre-processor itself, rather than by NJOY. This includes the processing of the $\mathrm{S}(\alpha, \beta)$ data to calculate cross section values and to generate the probability distributions to be used for sampling the $\alpha$ and $\beta$ values from which the secondary energies and angles are calculated. The methods used in BINGO are, for the most part, similar to those used in DICE [13], but with a number of improvements. The main enhancement has been to introduce the modelling of coherent elastic scattering, which occurs in crystalline materials and which was not treated in DICE. It is important, in particular, for thermal neutron scattering in graphite.

As in DICE, thermal neutron scattering in most nuclides can be treated by a free gas model, but the BINGO libraries include bound atom scattering data for a wider range of nuclides, to the extent that these are available in the evaluated nuclear data files.

\section{Validation}

MONK has a large validation database comprising MONK models for experimental configurations, based mainly upon
Table 1. Input parameters, default values and data range.

\begin{tabular}{lll}
\hline parameter & default & data range \\
\hline number of secondary energy bins & 32 & $8-1024$ \\
number of secondary angle bins & 128 & $8-1024$ \\
infinite dilution background & $1.0 \times 10^{10}$ & $1.0 \times 10^{5}-$ \\
cross section, barns & & $1.0 \times 10^{20}$ \\
subgroup generation tolerance, $\%$ & 0.01 & $1.0 \times 10^{-5}-1.0$ \\
subgroup relative width probability & 0.3 & $0.0-1.0$ \\
${ }^{238} \mathrm{U},{ }^{240} \mathrm{Pu}$ URR subgroup & 10 & $0.0-100.0$ \\
background cross section, barns & & \\
\hline
\end{tabular}

Table 2. List of nuclides.

\begin{tabular}{ll}
\hline nuclide & experiment \\
${ }^{234} \mathrm{U},{ }^{235} \mathrm{U},{ }^{238} \mathrm{U}$ & GODIVA \\
${ }^{239} \mathrm{Pu},{ }^{240} \mathrm{Pu},{ }^{241} \mathrm{Pu}, \mathrm{Ga}$ & JEZEBEL \\
${ }^{235} \mathrm{U},{ }^{238} \mathrm{U}$ & ZEBRA 8 \\
${ }^{1} \mathrm{H}, \mathrm{C},{ }^{16} \mathrm{O},{ }^{27} \mathrm{Al},{ }^{28} \mathrm{Si},{ }^{29} \mathrm{Si},{ }^{30} \mathrm{Si},{ }^{46} \mathrm{Ti},{ }^{47} \mathrm{Ti}$, & \\
${ }^{48} \mathrm{Ti},{ }^{49} \mathrm{Ti},{ }^{50} \mathrm{Ti}$, & \\
$\mathrm{V},{ }^{50} \mathrm{Cr},{ }^{52} \mathrm{Cr},{ }^{53} \mathrm{Cr},{ }^{54} \mathrm{Cr},{ }^{55} \mathrm{Mn},{ }^{54} \mathrm{Fe},{ }^{56} \mathrm{Fe},{ }^{57} \mathrm{Fe},{ }^{58} \mathrm{Fe}$, & \\
${ }^{58} \mathrm{Ni},{ }^{60} \mathrm{Ni},{ }^{61} \mathrm{Ni},{ }^{62} \mathrm{Ni},{ }^{64} \mathrm{Ni},{ }^{63} \mathrm{Cu},{ }^{65} \mathrm{Cu},{ }^{92} \mathrm{Mo},{ }^{94} \mathrm{Mo}$, & \\
${ }^{95} \mathrm{Mo},{ }^{96} \mathrm{Mo},{ }^{97} \mathrm{Mo},{ }^{98} \mathrm{Mo},{ }^{100} \mathrm{Mo}$ & \\
\hline
\end{tabular}

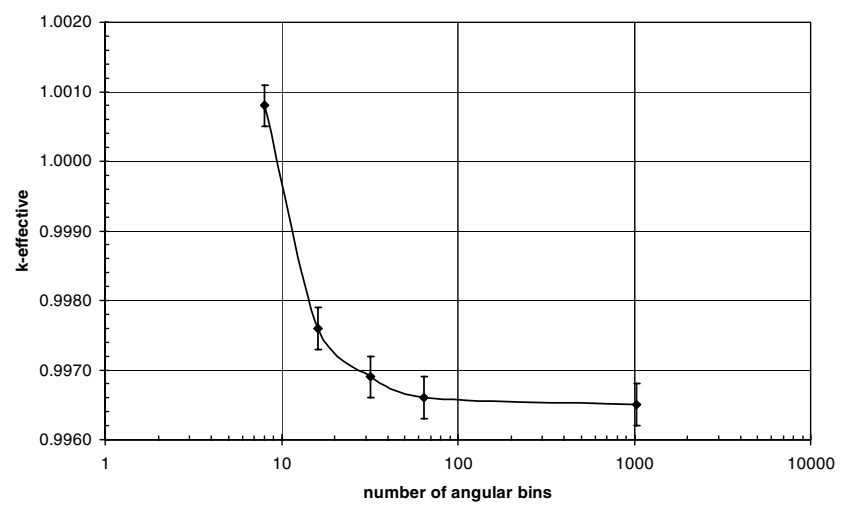

Fig. 1. Variation in the number of equiprobable angular bins for GODIVA.

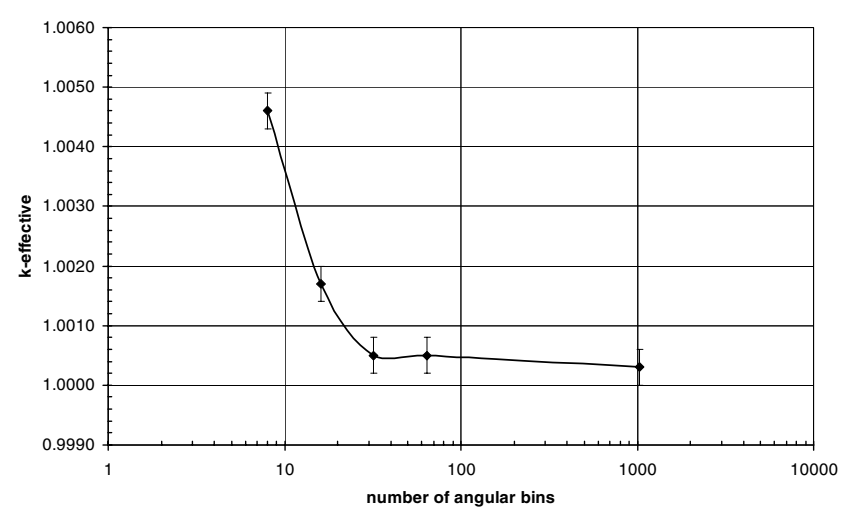

Fig. 2. Variation in the number of equiprobable angular bins for JEZEBEL. 


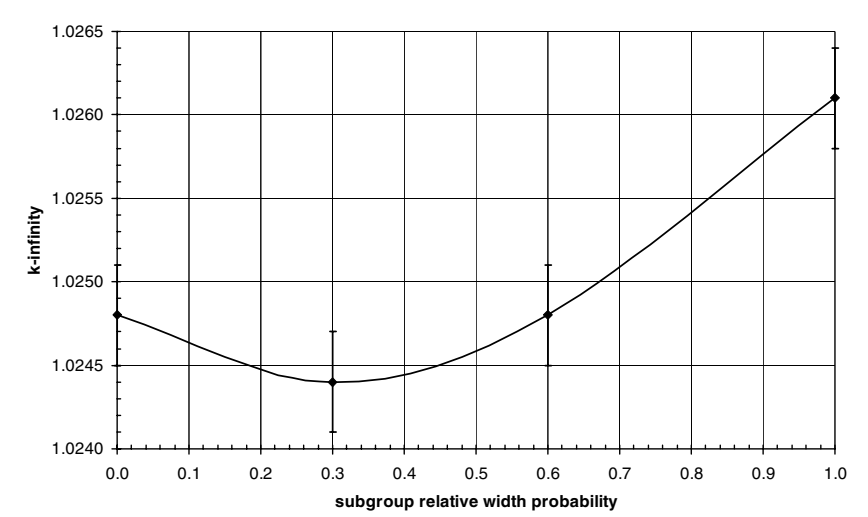

Fig. 3. Variation in the subgroup relative width probability for ZEBRA-8H.

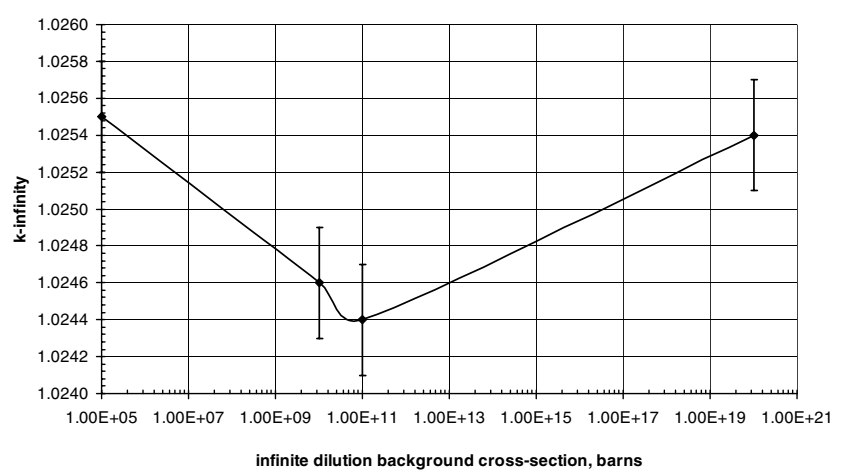

Fig. 4. Variation in the infinite background cross section for ZEBRA$8 \mathrm{H}$.

the specifications given in the handbook of the International Criticality Safety Benchmark Evaluation Project (ICSBEP) [14]. At present, there are 778 experimental configurations.

MONK has been fully validated against these experiments. This sensitivity study presents MONK validation results using JEFF 3.1.

This study examines only three experimental configurations, taken from the GODIVA [15], JEZEBEL [16] and ZEBRA 8 [17] experiments. These have been chosen so that the effect of changes to nuclear data parameter are emphasised. The experiments are described below.

\subsection{GODIVA - HEU-MET-FAST-001}

The GODIVA experiment was carried out at Los Alamos, USA in the 1950's to determine the critical mass of a bare uranium sphere enriched to 94 weight $\%{ }^{235} \mathrm{U}$. Full details of the experiment are given in ref. [15]. The simplified homogeneous spherical model is applied.

\subsection{JEZEBEL - PU-MET-FAST-001}

JEZEBEL refers to a series of critical experiments performed at Los Alamos, USA for over several years from the early 1950's onwards. The experiment considered here determined the critical mass of a sphere of alpha phase plutonium metal enriched to 95.2 weight $\%{ }^{239} \mathrm{Pu}$. The experiment is discussed in ref. [16].

\subsection{ZEBRA-8H - MIX-MET-FAST-008}

The ZEBRA reactor operated at Winfrith in the United Kingdom. The ZEBRA-8 experiment refers to $\mathrm{k}_{\infty}$ measurements made on assembly 8 in the 1960's. The measurements considered here examine assembly configuration $8 \mathrm{H}$, which consisted of a uranium metal plate enriched to 37.5 weight $\%$ ${ }^{235} \mathrm{U}$, sandwiched between natural uranium metal plates and contained in an alloy sheath. This is reflected axially and radially in the model. Full details are given in ref. [17].

\section{Sensitivity calculations}

The parameters chosen for this study are listed in table 1, together with their default values and the data range.

The nuclides considered in this study are given in table 2 .

\subsection{Methodology}

The BINGO pre-processor was run using the default values given in table 1 for each of the nuclides given in table 2 . These values were then perturbed. Only those nuclides with unresolved cross sections were subjected to the subgrouping treatment. For the non-fissile materials in ZEBRA-8, perturbations were also carried out on the coupled energy-angle data produced from file 6 [7] formatted data.

Standard MONK inputs were set up for each of the experiments. A matrix of runs was then executed for each experiment. In each case, default data were used, apart from specific perturbation under consideration.

\section{Results}

\subsection{GODIVA}

For GODIVA, K-effective values from MONK showed limited sensitivity, except to variations in the number of equiprobable angular bins. This is shown in figure 1 . The quoted experimental value [15] is $1.0000 \pm 0.0010$.

\subsection{JEZEBEL}

Again, for JEZEBEL, K-effective values from MONK showed limited sensitivity, except to variations in the number of equiprobable angular bins. This is shown in figure 2 . The quoted experimental value [16] is $1.0000 \pm 0.0020$.

\subsection{ZEBRA-8H}

The k-infinity MONK calculations for ZEBRA-8H showed sensitivity to both the subgroup relative width and the infinite dilution background cross section. This is shown in figures 3 and 4 . The quoted experimental value [17] is $1.0300 \pm 0.0025$. 


\section{Conclusions}

We have presented the results of a study to examine the sensitivity of the MONK criticality code to a variety of nuclear data input parameters.

In general, MONK was found to be insensitive to changes, particularly for the highly symmetric, fast systems seen in GODIVA and JEZEBEL. For these two experiments, MONK was seen to be sensitive to the number of equi-probable angular bins. At least 64 equiprobable angular bins should be used to describe secondary particles in the nuclear data used by MONK.

For ZEBRA-8H, MONK was seen to be sensitive to two of the unresolved resonance region parameters. These were the subgroup relative width and the infinite dilution background cross section. These results were somewhat unexpected and further work will be required.

The authors wish to thank the other members of the development team: David Bendall, Reg Brissenden, Andy Cooper, Richard Neal, Keith Searson (all British Nuclear Group Sellafield Ltd), Adam Bird, Pat Cowan and George Wright (all Serco Assurance).

\section{References}

1. N.R. Smith, M.J. Armishaw, A.J. Cooper, Current Status and Future Direction of the MONK Software Package, Proceedings of International Conference on Nuclear Criticality Safety, ICNC2003, Tokai-mura, Japan, Oct. 20-24 (2003).

2. N.R. Smith, M.J. Armishaw, J.L. Hutton, J. Sidell, The Unification of MONK - Extending the Monte Carlo Horizon, Proceedings of International Conference on Nuclear Criticality Safety, ICNC1999, Versailles, France, Sept. 20-24 (1999).

3. M.J. Armishaw, A.J. Cooper, Current Status and Future Direction of the MONK Software Package, Proceedings of the International Conference on Nuclear Criticality Safety, ICNC2007, St. Petersburg, Russia, May 28 - June (2007).
4. S.M. Connolly, M.J. Grimstone, The Development and Validation of a New Collision Processor for MONK, Proceedings of International Conference on Nuclear Criticality Safety, ICNC2003, Tokai-mura, Japan, Oct. 20-24 (2003).

5. J.B. Price, DICE Mk. V: The Preparation of Nuclear Data into a Form Suitable for Monte Carlo Calculations Using an Electronic Computer, AWRE 0-27/66 (United Kingdom Atomic Energy Authority, 1966).

6. J.S. Story, R.W. Smith, The 1981 Edition of the United Kingdom Nuclear Data Library: A Status Summary, DIDWG(81)/P246 (United Kingdom Atomic Energy Authority, 1981).

7. Members of the Cross Section Evaluation Working Group, ENDF- 6 Formats Manual - Data Formats and Procedures for the Evaluated Nuclear Data File ENDF/B-VI and ENDF/B-VII, edited by M. Herman, BNL-NCS-44945-05-Rev.

8. NEA Databank, The JEFF-3.1 Nuclear Data Library, JEFF Report 21 (OECD Nuclear Energy Agency, 2006).

9. A.J. Koning et al., The JEFF Evaluated Nuclear Data Project (this conference).

10. American National Standards Institute, American National Standard - Programming Language Fortran Extended, informally known as Fortran 90, (ANSI, Washington DC, 1997).

11. R.E. MacFarlane, D.W. Muir, The NJOY Nuclear Data Processing System, Version 91, LA-12740-M, 1994.

12. http://t2.lanl.gov/codes/njoy99/index.html.

13. D.E. Bendall, New Thermal Treatment for MONK, Paper II.1.6, Proceedings of International Conference on Nuclear Criticality Safety, ICNC1991, Oxford, United Kingdom, Sept. 9-13 (1991).

14. NEA Nuclear Science Committee, International Handbook of Evaluated Criticality Safety Benchmark Experiments, NEA/NSC/DOC/(95)03, 2006.

15. R.J. LaBauve, Bare, Highly Enriched Uranium Sphere (GODIVA), NEA/NSC/DOC/(95)03/II, 2002.

16. R.D. O'Dell, Bare Sphere of Plutonium-239 Metal (4.5 at.\%. 240Pu, $1.02 w t . \% \mathrm{Ga}$ ), NEA/NSC/DOC/(95)03/I, 2002.

17. D. Hanlon, K-Infinity Experiments in Fast/Intermediate Neutron Spectra For Various Fissile Materials, NEA/NSC/DOC/(95)03/VI, 1997. 